\title{
Computationally efficient parameter estimation and uncertainty quantification in complex physiological systems
}

\author{
Dr. Dirk Husmeier \\ University of Glasgow, UK
}

There have recently been impressive developments in the mathematical modelling of physiological processes. Under the research programme of the EPSRC-funded research centre SofTMech (http://www.softmech.org), we have developed mathematical models for the mechanical and electrophysiological processes of the heart, and the flow in the blood vessel network. This allows us to gain deeper insight into the state of a variety of serious cardiovascular diseases, like hypoxia (a condition in which a region of the body is deprived of adequate oxygen supply), angina (reduced blood flow to the heart), pulmonary hypertension (high blood pressure in the lungs) and myocardial infarction (heart attack). A more recent extension of this work to modelling blood flow in the eye also provides novel indicators to assess the degree of traumatic brain injury.

What all these models have in common is a complex mathematical description of the physiological processes in terms of differential equations that depend on various material parameters, related e.g. to the stiffness of the blood vessels or the contractility of the muscle fibres. While knowledge of these parameters would be of substantial benefit to the clinical practitioner to help them improve their diagnosis of the disease status, most of the parameters cannot be measured in vivo, i.e. in a living patient. For instance, the determination of the stiffness and contractility of the cardiac tissue would require the extraction of the heart from a patient and its inspection in a laboratory, which can only be done in a post mortem autopsy.

It is here that our mathematical models reveal their diagnostic potential. Our equations of the mechanical processes in the heart predict the movement of the heart muscle and how its deformations change in time. These movements can also be observed with magnetic resonance image (MRI) scans, and they depend on the physiological parameters. We can thus compare the predictions from our model with the patterns found in the MRI scans, and search for the parameters that provide the best agreement. In a previous proof-of-concept study we have demonstrated that the physiological parameters identified in this way lead to an improved understanding of the cardiac disease status, which is important for deciding on appropriate treatment options.

Unfortunately, the inference procedure described above faces enormous computational costs. We typically have a large number of physiological parameters, and an exhaustive search in a high-dimensional parameter space is a challenging problem. In addition, every time we change the parameters, our mathematical equations need to be solved again. This requires the application of complex numerical procedures, which take several minutes to converge. The consequence is that even with a high-performance computer, it takes several weeks to determine the physiological parameters in the way described above. It therefore appears that despite their enormous potential, state of the art mathematical modelling techniques can never be practically applied in the clinical practice, where diagnosis and decisions on alternative treatment option have to be made in real time.

In my talk, I will discuss how to address these difficulties with a statistical procedure. The idea is to approximate the computationally expensive mathematical model by a computationally cheap surrogate model called a statistical emulator. To create this emulator, we cover the parameter space with an appropriate design, solve the mathematical equations in parallel numerically for the chosen parameters, and then fit a non-linear statistical regression model to this training set. After this initial computational investment, the emulator thus created gives predictions for new parameter values practically instantaneously, allowing us to carry out the calibration procedure described above in real time. This will open the doors to harnessing the diagnostic potential of state-of-the art mathematical models for improved decision support in the clinic. 